\title{
Computerized Relational Agent to Deliver Alcohol Brief Intervention and Referral to Treatment in Primary Care: a Randomized Clinical Trial
}

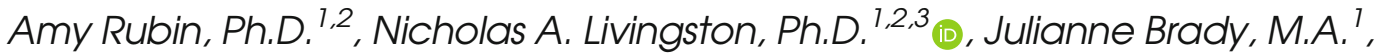 \\ Elise Hocking, Ph.D. ${ }^{1,2}$, Timothy Bickmore, Ph.D. ${ }^{4}$, Molly Sawdy, B.A. ${ }^{1}$, \\ Nancy Kressin, Ph.D. ${ }^{1,2}$, Richard Saitz, M.D., M.P.H. ${ }^{5}$, and Steven Simon, M.D., M.P.H. \\ 'U.S. Department of Veterans Affairs, VA Boston Healthcare System, 150 South Huntington Ave, Boston, MA, USA; ${ }^{2}$ Department of Psychiatry, Boston \\ University School of Medicine, Boston, MA, USA; ${ }^{3}$ Behavioral Sciences Division, National Center for PTSD, Boston, MA, USA; ${ }^{4}$ Computer Sciences, \\ Northeastern University, Boston, MA, USA; ${ }^{5}$ School of Public Health, Boston University, Boston, MA, USA; ${ }^{6}$ U.S. Department of Veterans Affairs, VA \\ Greater Los Angeles Healthcare System, Los Angeles, CA, USA; ${ }^{7}$ Harvard Medical School, Boston, MA, USA; ${ }^{8}$ David Geffen UCLA School of \\ Medicine, Los Angeles, CA, USA.
}

BACKGROUND: Alcohol screening and brief intervention have demonstrated efficacy but limited effectiveness and implementation in real-world primary care settings.

OBJECTIVE: To evaluate the effectiveness of a computerized Relational Agent programmed to provide alcohol screening, brief intervention, and referral to treatment. We hypothesized that participants in the experimental condition would report greater reductions in their drinking and higher rates of brief intervention and referrals to specialty care compared to those in treatment as usual (TAU).

DESIGN: This was a Hybrid I implementation design and stratified RCT. Participants were randomized to TAU or Relational Agent + TAU and assessed at baseline and 3month follow-up.

PARTICIPANTS: A total of 178 veteran participants were recruited by referral from primary care staff after a positive alcohol screen, or via letter sent do patients screening positive during recent visit.

INTERVENTION(S): TAU involved yearly reminders to screen alcohol use and provide brief intervention and treatment referrals, as needed. The Relational Agent added an automated brief intervention, a 1-month follow-up Relational Agent visit, and referral to treatment if needed.

MAIN MEASURES: We measured average drinks per day, drinking days per week, number of brief interventions, and number of referrals over 3 months.

KEY RESULTS: Participants decreased their drinking in both study conditions, with no significant between-group differences on primary alcohol measures. However, Relational Agent + TAU participants evidenced greater improvements regarding negative alcohol-related consequences over 3 months, and were significantly more likely to receive a brief intervention and referral to specialty care.

CONCLUSIONS: The Relational Agent successfully provided brief intervention and referred many more patients to specialty care and was able to intervene with patients with

Computerized Relational Agent in Primary Care

Received October 13, 2020

Accepted May 20, 2021

Published online June 18, 2021 less severe drinking without increasing primary care burden.

TRIAL REGISTRATION: clinicaltrials.gov, NCT02030288, https://clinicaltrials.gov/ct2/home

KEY WORDS: alcohol; primary care; technology; intervention; veterans.

J Gen Intern Med 37(1):70-7

DOI: $10.1007 / \mathrm{s} 11606-021-06945-9$

(C) This is a U.S. government work and not under copyright protection in the U.S.; foreign copyright protection may apply 2021

$\mathrm{U}$ nhealthy alcohol use remains a significant public health concern in the USA, ${ }^{1-3}$ and the vast majority of individuals who engage in unhealthy alcohol use never receive treatment. ${ }^{1,4}$ Unhealthy alcohol use is especially prevalent among veterans, ${ }^{5}$ who also evidence high rates of unmet treatment need. ${ }^{6,7}$ The Screening, Brief Intervention, and Referral to Treatment model (i.e., SBIRT) ${ }^{8}$ is the most prominent and widely adopted strategy for identifying patients who might be at risk for unhealthy alcohol use and for providing intervention and referral to specialty care within primary care settings. ${ }^{8-10}$ The efficacy of SBIRT in primary care is long established, ${ }^{9-11}$ yet barriers to implementation, fidelity, and small-modest effect sizes observed in effectiveness studies demonstrate the need for novel adaptations in order to improve patient outcomes.

The Veterans Health Administration (VHA) has mandated SBIRT across primary care clinics nationally, ${ }^{11}$ using automated clinical reminders, checklists, and performance standards to increase fidelity. Nevertheless, rates and quality of SBIRT are suboptimal, ${ }^{12-16}$ and little evidence for the effectiveness of these automated clinical reminders and brief interventions exists. ${ }^{13}, 16$ Significant practical barriers (e.g., competing clinical demands, limited staff support, and training) ${ }^{17-19}$ undermine SBIRT implementation, sustainability, and effectiveness.. 
To offset provider burden and facilitate implementation of SBIRT, it is critical to account for patient-level barriers, including concerns about confidentiality and fear of judgment from providers. ${ }^{20}$ Patients often experience discomfort discussing alcohol use with providers with whom they do not have a prior relationship, ${ }^{19,20}$ and stigma may drive reluctant to provide accurate alcohol use information, ${ }^{11}$ or engage in recommended treatment following alcohol use screening in primary care. ${ }^{20,}{ }^{21}$ Thus, innovation that seeks to address the existing screening, intervention, and referral gaps must also consider, and seek to reduce or circumvent, patient barriers.

Advances in automated intervention technologies could significantly improve alcohol use outcomes and treatment, ${ }^{22-}$ ${ }^{24}$ and computer-based Relational Agents represent a promising new line of intervention technology that can be easily implemented within real-world primary care settings. Relational Agents are designed to simulate face-to-face counseling, build rapport, and establish a therapeutic alliance, ${ }^{25}$ and can be tailored to respond to incoming information from patients in real-time. Relational Agent interventions have been shown to be effective in promoting behavior change, ${ }^{26}$ but have rarely been used to address substance use. ${ }^{27}$ However, previous research suggests that Relational Agent interventions are just as effective in screening for AUD as commonly used selfreport measures, ${ }^{28}$ and are well-accepted by patients. ${ }^{27}$

To bridge the gap between innovation and implementation and increase access to quality SBIRT intervention within primary care, we developed the first Relational Agent to deliver brief intervention and referral to treatment to veterans within VA primary care clinics. As part of this Hybrid Type I implementation trial, ${ }^{29}$ an initial sample of veterans participated in a three-phase RA development process to assist in the design and development of the Relational Agent. After participants completed the clinical trial, we conducted qualitative interviews about their experience (results reported separately).

The current paper presents the effectiveness trial results as compared to treatment as usual (TAU). In primary care TAU, patients are universally screened and receive brief intervention and referral if appropriate. We hypothesized that participants in the Relational Agent + TAU treatment condition would report greater reductions in average drinks per day and drinking days per week between baseline and 3-month follow-up. Secondarily, we hypothesized that rates of brief intervention and referral to treatment would be higher among Relational Agent + TAU recipients compared to TAU recipients. Lastly, we hypothesized that patients in the Relational Agent + TAU condition would report greater reductions in the percentage of heavy drinking days and negative alcohol consequences over time.

\section{METHODS}

\section{Participants}

Between August 2016 and August 2018, we assessed 1609 patients receiving care in three primary care clinics and two
Women's Health Clinics in one VHA healthcare system for eligibility. Some patients were referred directly to the study by primary care staff and others were invited by mail after being identified based on past 3-month positive alcohol screens via administrative data. A total of 1417 (88.1\%) were excluded from randomization, with 175 participants not meeting inclusion criteria and 1242 participants declining to participate. In total, 192 patients were enrolled and randomized to the Relational Agent + TAU intervention arm $(n=95)$ or TAU arm $(n=97)$. Fifteen were excluded from analyses due to mistakes in eligibility found after randomization, resulting in a final analytic sample of 178 patients (Fig. 1).

Eligibility criteria were as follows ${ }^{1}$ : a positive Alcohol Use Disorders Identification Test-C screening (AUDIT-C; $\geq 3$ for women and 4 for men $^{13}$ in the last 3 months), ${ }^{2}$ drinking above the NIAAA guidelines within the past 30 days (i.e., $>3 / 7$ standard drinks per day/week for women, $>4 / 14$ drinks per day/week for men), ${ }^{303}$ current stable phone number and address for follow-up, and ${ }^{4}$ willingness to provide contact information for at least one "locator" (i.e., a person who always knows their whereabouts for follow-up). Exclusion included receipt of substance use treatment in the past 30 days or participation in the usability and feedback part of the study.

\section{Procedure}

All study procedures and documents were approved by the [VA Boston Healthcare System Institutional Review Board] Institutional Review Board. Participants were stratified by baseline alcohol use severity, i.e., hazardous drinking vs. having an AUD based on screening with the Multiphasic International Neuropsychiatric Interview (MINI) - Alcohol Scale $^{31}$ and/or previous substance abuse treatment, and gender, and then randomized into TAU or Relational Agent + TAU. Participants completed a follow-up survey at 3 months.

Baseline Interview. Baseline demographic data, past 30-day alcohol consumption (Quick Drinking Screen, QDS), ${ }^{32,33}$ recent negative consequences associated with their drinking (Short Inventory of Problems, SIP),${ }^{34}$ and completed MINI were collected. Participants randomized to the Relational Agent + TAU group were immediately introduced to the Relational Agent.

Follow-up Interview. Three months after the baseline interview, participants were contacted by phone, and the Quick Drinking Screen and Short Inventory of Problems were administered.

Data Collection via Clinical Data Warehouse and Chart Review. For patient encounter information, such as brief intervention received and referrals made, we used data collected and stored by the Relational Agent software, as well as recorded by primary care providers (PCPs) or PCP staff within patients' electronic health records during their inperson visits. For administrative chart data, brief intervention 


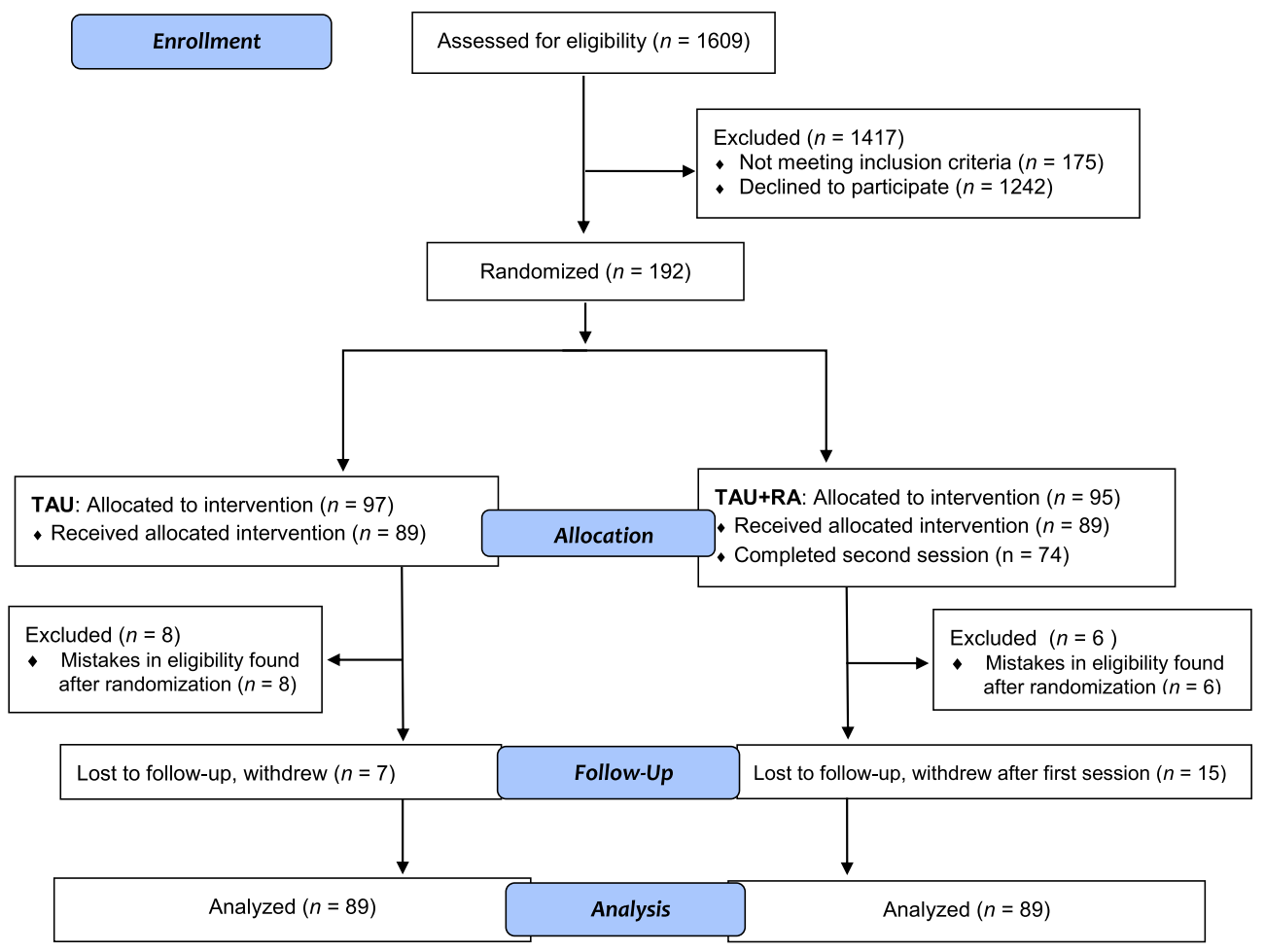

Fig. 1 CONSORT diagram.

was defined as any documented interaction in which the provider counseled the patient to reduce or abstain from drinking. For PCP referral data, we reviewed patient records for consults/referrals submitted by the provider/clinic to any outpatient or residential specialty substance use disorder clinics. ${ }^{35}$

\section{Intervention}

Treatment as Usual (TAU). Patients were screened annually by the nurse or health tech for alcohol problems using the 3item AUDIT-C. ${ }^{36}$ PCPs are alerted through the electronic medical record if the patient screens positive. PCPs are prompted to provide a brief intervention using a prepared checklist of items, and then prompted to refer patients who score 8 or above to specialty care. Patients were recruited to the study after this process concluded.

\section{Relational Agent Plus Treatment as Usual (Relational Agent} $+\boldsymbol{T A U}$ ). Participants met with the Relational Agent immediately after the baseline interview, and again for a follow-up Relational Agent visit in primary care 1 month later. The Relational Agent was programmed to speak with patients, who respond by choosing buttons containing phrases on a touch screen (see Fig. 2). The Relational Agent administered the full AUDIT because additional information was needed to conduct the brief intervention. It was designed to deliver the same kind of care as the PCP would ideally deliver and to conform to SBIRT procedures. The brief intervention was based on Motivational Interviewing strategies, ${ }^{37}$ such as eliciting concern about consequences of drinking, bolstering motivation (e.g., importance, confidence, and readiness rulers; decisional balance exercise), and providing reflections and summaries of the interaction. At the end, the Relational Agent asked for a commitment to change. If the participant screened positive for alcohol problems, the Relational Agent offered a treatment referral and generated a report for the patient. The consult to specialty care was placed by the first author.

\section{Analysis Plan}

Data Handling and Analytic Strategy. Independent variables included time, treatment condition, and treatment condition by time; model covariates included gender and alcohol severity at baseline to control for known bias in the results. Average drinks per day and drinking days per week were modeled as count variables; percentage of heavy drinking days and alcohol consequences (Short Inventory of Problems (SIP)) were square root and log transformed (base $e$ ), respectively. We tested hypotheses using generalized and linear mixed modeling procedures with repeated measures nested within participant ID. We specified hierarchical linear models and restricted maximum likelihood (REML) for percentage of heavy drinking days and SIP scores and hierarchical Poisson regression models, log link, and maximum likelihood for average drinks per day and drinking days per week outcomes. Each model included fixed effects with a random effect specified for participant ID to account for participant-level variability. We analyzed brief intervention and referral to treatment between conditions using chi-square tests. 


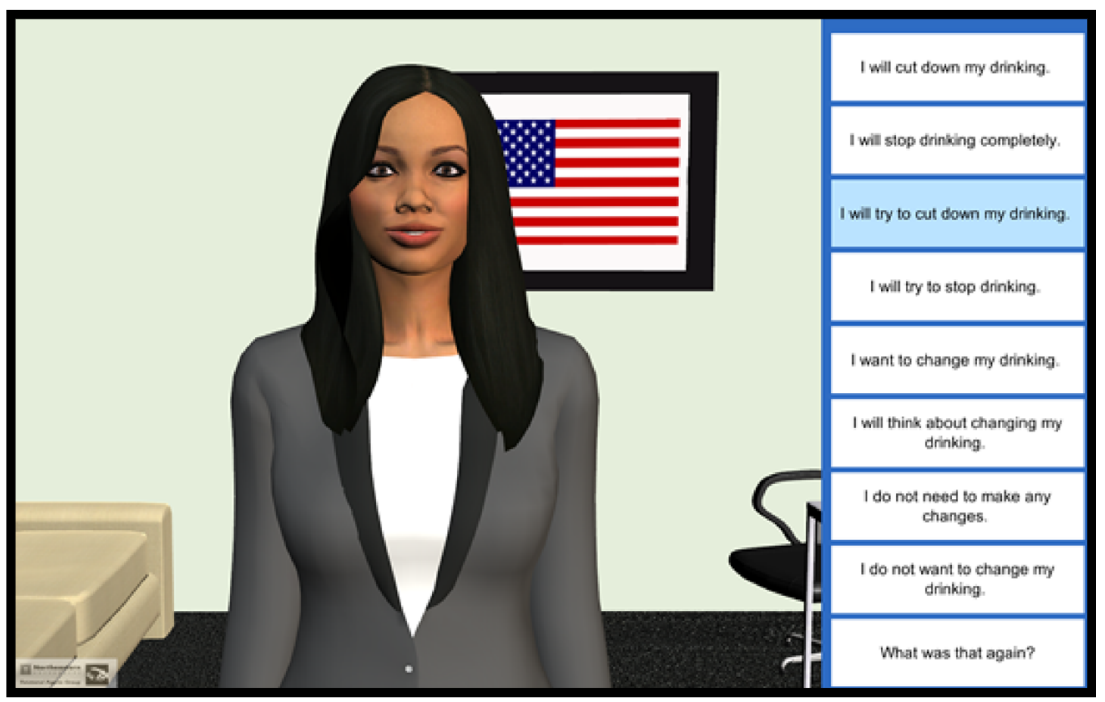

Fig. 2 Screenshot of Laura.

Data were analyzed in R using base packages ${ }^{38}$ and the lme $4^{39}$ and nlme ${ }^{40}$ packages. We also performed chi-square and $t$ tests to evaluate differences between treatment conditions regarding categorical and continuous variables, respectively (Table 1).

\section{RESULTS}

\section{Descriptive Results}

Participants were $89 \%$ male and $67 \%$ White and $89 \%$ completed the 3-month follow-up. We found no statistically significant differences between study groups on demographics but some regarding baseline alcohol scores (see Table 1).

\section{Primary Hypothesis}

Average Drinks per Day. Based on generalized mixed model results, participants in both conditions reported significant decreases in average drinks per day over 3 months $(b=-.39, z=-5.79, p<.001)$; with no differences between groups $(b=-.11, z=-.84, p=$ .39) (see Fig. 3; Supplemental Table 1). Women reported lower average drinking compared to men $(b=$ $-.67, z=-3.37, p<.001)$. Participants in the Relational Agent + TAU condition reported less average daily drinking $(b=-.31, z=-2.72, p=.006)$; and participants with AUD reported higher average daily drinking $(b=.33, z=2.36, p=.01)$.

Drinking Days per Week. Participants reported significant reductions in drinking days per week $(b=-.21, z=$ $-3.84, p<.001$ ), but similar rates of change between treatment conditions $(b=-.01, z=-.16, p=.87$ ) (see Fig. 3; Supplemental Table 1). Interestingly, participants
Table 1 Baseline Characteristics of the Sample

\begin{tabular}{|c|c|c|c|}
\hline & $\begin{array}{l}\text { Relational Agent } \\
+ \text { TAU }\end{array}$ & TAU & \\
\hline & $M(S D)$ & $M(S D)$ & $p(t)$ \\
\hline Age & $49.52(17.34)$ & $\begin{array}{l}53.90 \\
(18.39)\end{array}$ & ns \\
\hline \multirow[t]{2}{*}{ Years of education } & $14.46(2.31)$ & $\begin{array}{l}14.83 \\
(2.38)\end{array}$ & ns \\
\hline & $n(\%)$ & $n(\%)$ & $\begin{array}{l}p \\
\left(\chi^{2}\right)\end{array}$ \\
\hline Gender & & & ns \\
\hline Male & $79(88.76 \%)$ & $\begin{array}{l}79 \\
(88.76 \%)\end{array}$ & \\
\hline Female & $10(11.24 \%)$ & $\begin{array}{l}10 \\
(11.24 \%)\end{array}$ & \\
\hline Race/ethnicity & & & ns \\
\hline White & $60(67.42 \%)$ & $\begin{array}{l}60 \\
(67.42 \%)\end{array}$ & \\
\hline Hispanic/Latina/o & $4(4.49 \%)$ & $7(7.87 \%)$ & \\
\hline $\begin{array}{l}\text { Black/African Ameri- } \\
\text { can }\end{array}$ & $19(21.35 \%)$ & $\begin{array}{l}14 \\
(15.73 \%)\end{array}$ & \\
\hline Native American/ & $3(3.37 \%)$ & $0(0 \%)$ & \\
\hline \multicolumn{4}{|l|}{ Alaskan Native } \\
\hline Asian & $1(1.12 \%)$ & $0(0 \%)$ & \\
\hline Multi-racial & $1(1.12 \%)$ & $3(3.37 \%)$ & \\
\hline Other & $1(1.12 \%)$ & $3(3.37 \%)$ & \\
\hline Unknown/not disclosed & $0(0 \%)$ & $2(2.25 \%)$ & \\
\hline Employment & & & ns \\
\hline Full-time & $35(39.32 \%)$ & $\begin{array}{l}30 \\
(33.70 \%)\end{array}$ & \\
\hline Part-time & $8(9.0 \%)$ & $4(5.50 \%)$ & \\
\hline Unemployed & $4(5.50 \%)$ & $5(5.60 \%)$ & \\
\hline In-school & $6(6.74 \%)$ & $6(6.74 \%)$ & \\
\hline Disability & $21(23.60 \%)$ & $\begin{array}{l}23 \\
(25.84 \%)\end{array}$ & \\
\hline Retired & $15(16.86 \%)$ & $\begin{array}{l}21 \\
(23.60 \%)\end{array}$ & \\
\hline $\begin{array}{l}\text { Alcohol-related } \\
\text { measures }\end{array}$ & $n(\%)$ & $n(\%)$ & $\begin{array}{l}p \\
\left(\chi^{2}\right)\end{array}$ \\
\hline \multicolumn{4}{|l|}{ MINI } \\
\hline Yes & 69 & 70 & ns \\
\hline \multirow[t]{2}{*}{ No } & 20 & & \\
\hline & $M(S D)$ & $M(S D)$ & $p(t)$ \\
\hline Average drinks per day & 2.98 & 4.00 & .03 \\
\hline Drinking days per week & 4.07 & 4.71 & .03 \\
\hline $\begin{array}{l}\text { Percentage of heavy } \\
\text { drinking days }\end{array}$ & .27 & .31 & ns \\
\hline $\begin{array}{l}\text { Short Inventory of } \\
\text { Problems (SIP) }\end{array}$ & 9.38 & 8.84 & ns \\
\hline
\end{tabular}



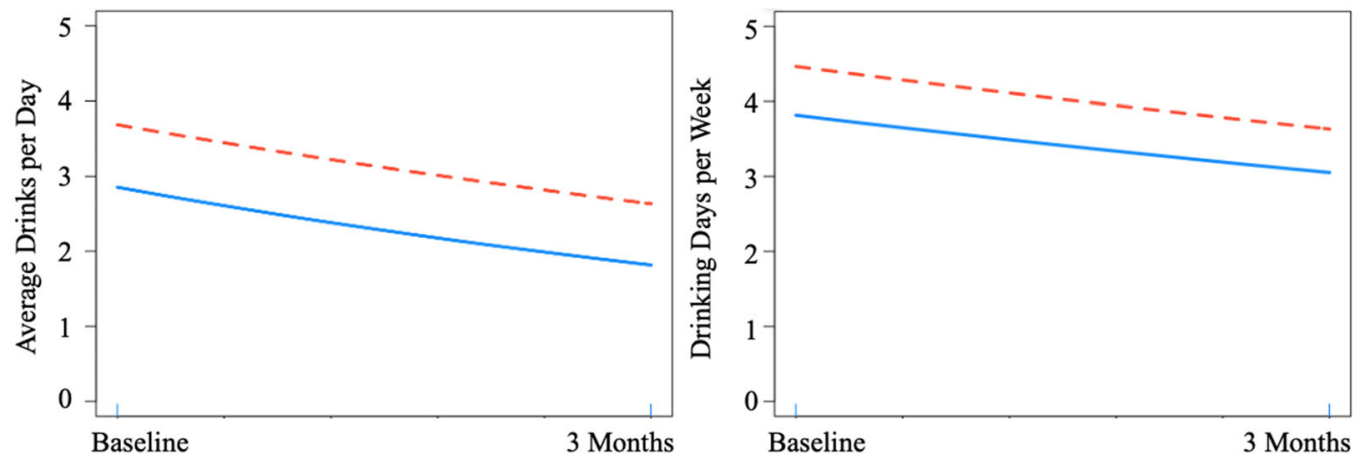

TAU
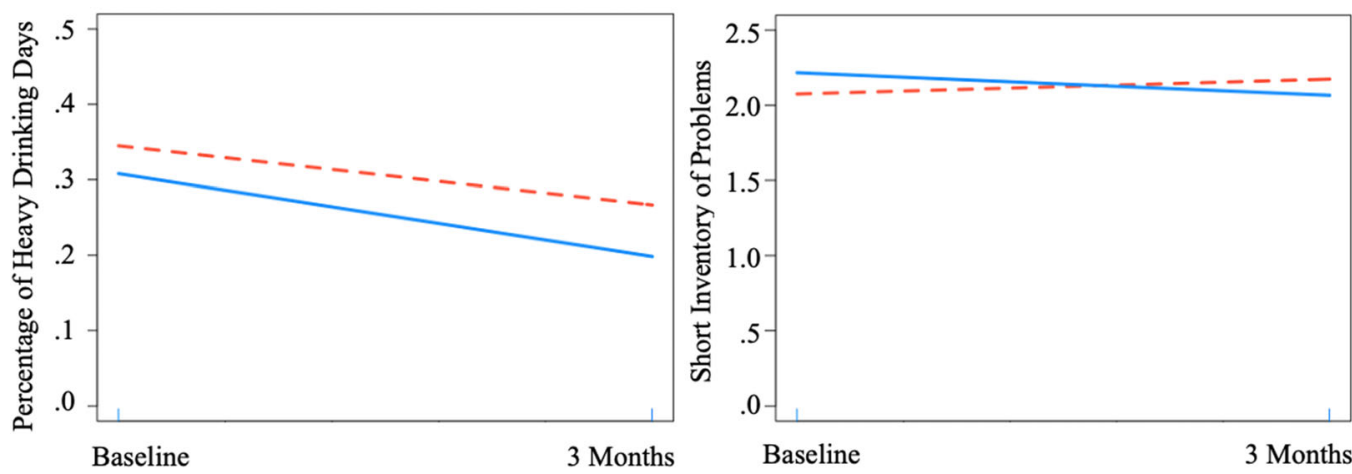

$\mathrm{RA}+\mathrm{TAU}$

Fig. 3 Model-implied trajectories of change from baseline to 3-month follow-up for primary, secondary, and exploratory outcome models.

with AUD at baseline reported fewer drinking days on average over the study period $(b=-.16, z=-1.98, p=$ .04). Relational Agent + TAU participants also reported fewer drinking days on average $(b=-.16, z=-2.34, p$ $=.01)$.

\section{Secondary Hypotheses}

Brief Intervention and Referral. All participants in the Relational Agent + TAU condition received brief intervention via the Relational Agent platform, compared to $43.82 \%(n=39)$ from their PCP providers in the TAU condition $\left(\chi^{2}=66.78, p<.001\right)$. Examining only those with AUDIT-C scores at or above 5 (cutoff used for VA providers), results still favored the Relational Agent condition $\left(100 \%\right.$ vs. $33.33 \%$; $\left.\chi^{2}=11.31, p<.001\right)$. Twentyfive participants received a referral to specialty substance use disorder treatment: 24 in the Relational Agent + TAU condition, and one $(<1 \%)$ from their PCP in the TAU condition $\left(\chi^{2}=21.24, p<.001\right)$. Seventy-one percent of participants attended the referral appointment. Since referral decisions in the VA are based on automated reminders, which are triggered for anyone with an AUDIT-C of 8 or higher, we performed additional analyses for the 42 patients ( 21 per condition) who scored at or above this cutoff. We found that $28.57 \%$ (6 out of 21 remaining) of these participants in the Relational Agent + TAU condition and $4.76 \%$ ( 1 out of 21 ) in the TAU condition received a referral $\left(\chi^{2}=2.74, p=.098\right)$.

\section{Exploratory Hypotheses}

Percentage of Heavy Drinking Days. In our linear mixed model, the percentage of heavy drinking days decreased over time $(b=-.07, z=-4.81, p<.001)$, with similar rates of change between conditions $(b=-.02, z=-.79, p=.42$ ) (see Fig. 3; Supplemental Table 1). Participants with suspected AUD reported a greater percentage of heavy drinking on average $(b=.11, z=3.46, p<.001)$.

Short Inventory of Problems. Total scores were higher, on average, among those with heavier baseline alcohol use $(b=$ $1.21, t=8.87, p<.001)$. In our linear mixed model, rates of change were greater for participants in the Relational Agent + TAU condition $(b=-.02, t=-2.25, p=.02)$ (see Fig. 3; Supplemental Table 1).

\section{DISCUSSION}

In this study, we created a Relational Agent to provide alcohol screening, brief intervention, and referral to treatment to patients within primary care. While participants in both TAU and Relational Agent + TAU condition improved their drinking, we did not observe accelerated improvement among participants in the Relational Agent + TAU condition over 3 months. This may be an artifact of greater drinking in the TAU group despite randomization. Heavier drinkers would be expected to decrease their drinking more rapidly than lighter drinkers just based on regression to the mean. However, participants in the Relational Agent + 
TAU condition were significantly more likely to receive brief intervention and referrals for follow-up specialty substance use treatment, and also reported greater improvements in alcohol use-related negative consequences (SIP) by 3 months. This suggests that the Relational Agent can both decrease negative consequences and increase referrals to needed treatment.

The observed benefits of the Relational Agent intervention should be viewed in light of the fact that the Relational Agent can provide SBIRT without burdening primary care staff, and could be administered in waiting rooms or at home. The timesaving potential of Relational Agent interventions could lead to more consistent and thorough assessment of alcohol use, because the Relational Agent administers the full-length version of the AUDIT or additional screening measures without placing more administrative burden on PCP providers or clinic staff.

Use of Relational Agent interventions could also lead to a more standardized application of SBIRT procedures. Previous research indicates that AUDIT-C scores differ significantly depending on who administers the measure and how, ${ }^{14}$ and patients may be more open to discussing alcohol use with a Relational Agent. Anecdotally, we observed a few cases where the AUDIT-C was inadvertently administered more than once during the same visit by different staff, with different results. When administered by the Relational Agent, patients may answer questions in a more consistent manner, which might help overcome assessment and intervention barriers (e.g., stigma) ${ }^{12}$ and bridge service gaps without increasing PCP or clinic burden.

In addition, all participants who were assigned to the Relational Agent + TAU condition received brief intervention, as compared to just under $44 \%$ of participants in the TAU condition. The Relational Agent also referred a much higher proportion of patients to treatment, with $71 \%$ of referrals resulting on a first visit to the clinic. While VA PCPs are recommended to offer referrals to patients scoring an 8 or higher on the AUDIT-C, the Relational Agent was not constrained in this way, nor by the myriad of other tasks that PCPs must perform during patient encounters. Thus, our finding that Relational Agent provided significantly more referrals overall is an important outcome for patients in this study, considering that the majority of patients who present with AUD in a primary care setting may not have received a referral otherwise.

Despite similar rates of change in alcohol use across both conditions, patients in the Relational Agent + TAU condition reported fewer alcohol-related problems 3 months after intervention. The lack of intervention effect on primary alcohol measures may relate to the significantly higher baseline and scores among TAU participants, but not alcohol consequences. It is possible that Relational Agent + TAU participants were successful in implementing protective behavioral strategies that prevented negative consequences of their drinking, without detectible differences relative to TAU patients on primary alcohol consumption measures. This is worth future study, as harm-reduction and reduction of negative consequences is by itself a viable intervention target. ${ }^{41}$

\section{Limitations}

The current study was limited in that eligibility was determined by, and recruitment was contingent on, AUDIT-C scores obtained by clinic staff who appear to have administered the measure in an inconsistent manner. Generalizability of our results should be interpreted in light of underrepresentation of women, which is expected in the male-dominated veteran population, and racial and ethnic diversity.

\section{CONCLUSION}

The persistently high prevalence of hazardous drinking and AUD in general and patients in particular suggests that innovation is needed that circumvents persistent barriers to treatment and recovery. Relational Agent technology has the potential to engage patients efficiently and in a standardized way. In addition to reducing provider burden, Relational Agent interventions could address or circumvent entirely patient barriers to care, including stigma associated with disclosing alcohol use and treatment seeking. ${ }^{11,19,20}$ It would be possible now to develop Relational Agent interventions to deliver SBIRT to patients through their own electronic device in their own home, thus increasing privacy and eliminating travel burden associated with in-person care, and increasing efficiency with respect to patient flow within VHA primary care clinics. This is an especially appealing advantage of Relational Agent technology in light of recent social distancing measures in response to COVID-19. Extensions of this technology could improve clinic efficiency and AUD treatment engagement, but also offset risk associated with clinic closures or inperson treatment restrictions in response to ongoing or future pandemic restrictions. Though we did not find that adding the Relational Agent to intervention by PCP staff improved drinking outcomes, the Relational Agent did deliver brief intervention and referrals to treatment more frequently to patients presenting with a wider range of AUDIT-C scores compared to VA primary care providers. As such, Relational Agent interventions might prove to be important tools to help address AUD concerns remotely, in a structured yet adaptable manner that could be scaled to increase efficiency of clinic operations, decrease provider burden, and potentially improve treatment access.

Corresponding Author: Nicholas A. Livingston, Ph.D.; U.S. Department of Veterans Affairs, VA Boston Healthcare System, 150 South Huntington Ave, Boston, MA 02130, USA (e-mail: nicholas. livingston@va.gov).

Supplementary Information The online version contains supplementary material available at https://doi.org/10.1007/s11606-02106945-9.

Funding This research was supported by the Department of Veterans Affairs, Veterans Health Administration, Health Services Research and Development (HSR\&D) Services (IIR11-3346; PI Simon). 


\section{Declarations:}

Conflict of Interest: The authors declare that they do not have a conflict of interest.

\section{REFERENCES}

1. Substance Abuse and Mental Health Services Administration. Key substance use and mental health indicators in the United States: results from the 2018 National Survey on Drug Use and Health (HHS Publication No. PEP19-5068, NSDUH Series H-54). Rockville, MD: Center for Behavioral Health Statistics and Quality. Substance Abuse and Mental Health Services Administration, Retrieved from https://www samhsa gov/data. 2020.

2. Grant BF, Goldstein RB, Saha TD, et al. Epidemiology of DSM-5 alcohol use disorder: results from the National Epidemiologic Survey on Alcohol and Related Conditions III. JAMA psychiatry. 2015;72(8):757-766.

3. National Institute on Alcohol Abuse and Alcoholism. Alcohol Facts and Statistics. Updated February 2020. Accessed August 15, 2020. https:// www.niaaa.nih.gov/publications/brochures-and-fact-sheets/alcoholfacts-and-statistics

4. Thompson A, Ashcroft DM, Owens L, van Staa TP, Pirmohamed M. Drug therapy for alcohol dependence in primary care in the UK: a Clinical Practice Research Datalink study. PloS one. 2017;12(3)

5. Lan CW, Fiellin DA, Barry DT, et al. The epidemiology of substance use disorders in US veterans: a systematic review and analysis of assessment methods. The American Journal on Addictions. 2016;25(1):7-24.

6. Golub A, Vazan P, Bennett AS, Liberty HJ. Unmet need for treatment of substance use disorders and serious psychological distress among veterans: a nationwide analysis using the NSDUH. Military medicine. 2013;178(1): 107-114.

7. Hoge C, Messer S, Castro C. Combat duty in Iraq and Afghanistan mental health problems. New England Journal of Medicine. 2004;351(17):17981800 .

8. Babor TF, McRee BG, Kassebaum PA, Grimaldi PL, Ahmed K, Bray J. Screening, Brief Intervention, and Referral to Treatment (SBIRT): Toward a Public Health Approach to the Management of Substance Abuse. Substance Abuse. 2007;28(3):7-30. doi:https://doi.org/10.1300/ J465v28n03_03

9. Agerwala SM, McCance-Katz EF. Integrating Screening, Brief Intervention, and Referral to Treatment (SBIRT) into Clinical Practice Settings: a Brief Review. Journal of Psychoactive Drugs. 2012;44(4):307-317. doi:https://doi.org/10.1080/02791072.2012.720169

10. Kaner EF, Beyer F, Dickinson HO, et al. Effectiveness of Brief Alcohol Interventions in Primary Care Populations. Cochrane Database Systematic Review. 2007;(2):CD004148. doi:https://doi.org/10.1002/ 14651858.CD004148.pub3

11. Teeters JB, Lancaster CL, Brown DG, Back SE. Substance Use Disorders in Military Veterans: Prevalence and Treatment Challenges. Substance Abuse and Rehabilitation. 2017;8:69-77. doi:https://doi.org/10.2147/ SAR.S116720

12. Lapham GT, Achtmeyer CE, Williams EC, Hawkins EJ, Kivlahan DR, Bradley KA. Increased Documented Brief Alcohol Interventions with a Performance Measure and Electronic Decision Support. Medical Care. Feb 2012;50(2):179-87. doi:https://doi.org/10.1097/MLR. Ob013e3181e35743

13. Williams EC, Lapham G, Achtmeyer CE, Volpp B, Kivlahan DR, Bradley KA. Use of an electronic clinical reminder for brief alcohol counseling is associated with resolution of unhealthy alcohol use at follow-up screening. Journal of General Internal Medicine. 2010;25(1):11-17.

14. Bradley KA, Lapham GT, Hawkins EJ, et al. Quality Concerns with Routine Alcohol Screening in VA Clinical Settings. Journal of General Internal Medicine. Mar 2011;26(3):299-306. doi:https://doi.org/10. 1007/s11606-010-1509-4

15. Bradley KA, Williams EC, Achtmeyer CE, et al. Measuring Performance of Brief Alcohol Counseling in Medical Settings: a Review of the Options and Lessons from the Veterans Affairs (VA) Health Care System. Substance Abuse. 2007;28(4):133-49. doi:https://doi.org/10.1300/J465v28n04_ 05

16. Williams EC, Rubinsky $\mathrm{AD}$, Chavez $\mathrm{LJ}$, et al. An early evaluation of implementation of brief intervention for unhealthy alcohol use in the US Veterans Health Administration. Addiction. 2014;109(9):1472-1481.

17. Osborn R, Moulds D, Schneider EC, Doty MM, Squires D, Sarnak DO. Primary care physicians in ten countries report challenges caring for patients with complex health needs. Health Affairs. 2015;34(12):21042112.

18. Mertens JR, Chi FW, Weisner CM, et al. Physician versus nonphysician delivery of alcohol screening, brief intervention and referral to treatment in adult primary care: the ADVISe cluster randomized controlled implementation trial. Addiction Science \& Clinical Practice. 2015;10(1):26.

19. Williams EC, Achtmeyer CE, Young JP, et al. Local Implementation of Alcohol Screening and Brief Intervention at Five Veterans Health Administration Primary Care Clinics: Perspectives of Clinical and Administrative Staff. Journal of Substance Abuse Treatment. Jan 2016;60:2735. doi:https://doi.org/10.1016/j.jsat.2015.07.011

20. McNeely J, Kumar PC, Rieckmann T, et al. Barriers and facilitators affecting the implementation of substance use screening in primary care clinics: a qualitative study of patients, providers, and staff. Addiction Science \& Clinical Practice. 2018;13(1):8.

21. Probst C, Manthey J, Martinez A, Rehm J. Alcohol use disorder severity and reported reasons not to seek treatment: a cross-sectional study in European primary care practices. Substance Abuse Treatment, Prevention, and Policy. 2015;10(1):32.

22. Livingston NA, Mahoney CT, Ameral V, et al. Changes in alcohol use, PTSD hyperarousal symptoms, and intervention dropout following veterans' use of VetChange. Addictive behaviors. 2020:106401.

23. Livingston NA, Shingleton R, Heilman ME, Brief D. Self-help smartphone applications for alcohol use, PTSD, anxiety, and depression: addressing the new research-practice gap. Journal of Technology in Behavioral Science. 2019;4(2):139-151.

24. Ashford RD, Bergman BG, Kelly JF, Curtis B. Systematic review: digital recovery support services used to support substance use disorder recovery. Human Behavior and Emerging Technologies. 2020;2(1):18-32.

25. Bickmore TW, Caruso L, Clough-Gorr K, Heeren T. 'It's Just Like You Talk to a Friend' Relational Agents for Older Adults. Interact Comput. 12// 2005; 17(6):711-735. doi:10.1016/j.intcom.2005.09.002

26. Bickmore TW, Silliman RA, Nelson K, et al. A randomized controlled trial of an automated exercise coach for older adults. Journal of the American Geriatrics Society. 2013;61(10):1676-1683.

27. Auriacombe M, Moriceau S, Serre F, et al. Development and Validation of a Virtual Agent to Screen Tobacco and Alcohol Use Disorders. Drug and alcohol dependence. Dec 1 2018;193:1-6. doi:https://doi.org/10.1016/j. drugalcdep.2018.08.025

28. Bickmore T, Rubin A, Simon SR. Substance use screening using virtual agents: towards automated Screening, Brief Intervention, and Referral to Treatment (SBIRT). ACM International Conference on Intelligent Virtual Agents (IVA). 2020;

29. Curran GM, Bauer M, Mittman B, Pyne JM, Stetler C. Effectivenessimplementation hybrid designs: combining elements of clinical effectiveness and implementation research to enhance public health impact. Medical care. 2012;50(3):217.

30. National Institute on Alcohol Abuse and Alcoholism. Drinking Levels Defined. U.S. Department of Health and Human Services. 2020. https:// www.niaaa.nih.gov/alcohol-health/overview-alcohol-consumption/moderate-binge-drinking

31. Sheehan DV, Lecrubier Y, Sheehan KH, et al. The Mini-International Neuropsychiatric Interview (MINI): the development and validation of a structured diagnostic psychiatric interview for DSM-IV and ICD-10. Journal of Clinical Psychiatry. 1998.

32. Sobell LC, Agrawal S, Sobell MB, et al. Comparison of a quick drinking screen with the timeline followback for individuals with alcohol problems. J Stud Alcohol. Nov 2003;64(6):858-61.

33. Roy M, Dum M, Sobell LC, et al. Comparison of the quick drinking screen and the alcohol timeline followback with outpatient alcohol abusers. Substance Use \& Misuse. Dec 2008;43(14):2116-23. doi:90318344110.1080/10826080802347586

34. Miller WR, Tonigan JS, Longabaugh R. The Drinker Inventory of Consequences (DrInC): an instrument for assessing adverse consequences of alcohol abuse. Vol. 4. 1995. Project MATCH Monograph Series.

35. Saunders JB, Aasland OG, Babor TF, de la Fuente JR, Grant M. Development of the Alcohol Use Disorders Identification Test (AUDIT): WHO collaborative project on early detection of persons with harmful alcohol consumption-II. Addiction. Jun 1993;88(6):791-804.

36. Bush K, Kivlahan DR, McDonell MB, Fihn SD, Bradley KA. The AUDIT alcohol consumption questions (AUDIT-C): an effective brief screening test for problem drinking. Ambulatory Care Quality Improvement Project (ACQUIP). Alcohol Use Disorders Identification Test. Archives of Internal Medicine. Sep 14 1998;158(16):1789-95. 
37. Miller WR, Rollnick S. Motivational Interviewing: Preparing People for Change. 2nd ed. Guilford Publications; 2002:428.

38. Team RC. R: A language and environment for statistical computing. Vienna, Austria; 2013.

39. Bates D, Mächler M, Bolker B, Walker S. Fitting linear mixed-effects models using lme4. arXiv preprint arXiv:14065823. 2014;
40. Pinheiro J, Bates D, DebRoy S, Sarkar D. R Core Team (2019). nlme: linear and nonlinear mixed effects models. R package version 3.1-148. 2020.

41. Witkiewitz K, Heather N, Falk DE, et al. World Health Organization risk drinking level reductions are associated with improved functioning and are sustained among patients with mild, moderate and severe alcohol dependence in clinical trials in the United States and United Kingdom. Addiction. 2020.

Publisher's Note Springer Nature remains neutral with regard to jurisdictional claims in published maps and institutional affiliations. 